\title{
Life after the telescope conjecture
}

[To appear in Algebraic K-theory and Algebraic Topology, edited by R. F. Jardine, Kluwer Academic Publishers]

\author{
Douglas C. Ravenel \\ University of Rochester \\ Rochester, New York $14627^{*}$
}

October 23, 1992

\begin{abstract}
We discuss the chromatic filtration in stable homotopy theory and its connections with algebraic K-theory, specifically with some results of Thomason, Mitchell, Waldhausen and McClure-Staffeldt. We offer a new definition (suggested by the failure of the telescope conjecture) of the chromatic filtration, in which all of the localization functors used are finite.
\end{abstract}

The telescope conjecture ( 2.2 below) was first announced by the author in 1977, published in [Rav84], and disproved in 1990 [Rav92b] [Rav]. It is a statement about the chromatic filtration, which was originally devised to understand the stable homotopy theory of finite complexes. Very briefly, for each prime there is a series of localization functors $L_{n}$ defined in the homotopy category (both stably and unstably) so that for each spectrum $X$ there is an inverse system (called the chromatic tower)

$$
L_{0} X \longleftarrow L_{1} X \longleftarrow L_{2} X \longleftarrow \cdots
$$

The chromatic filtration of $\pi_{*}(X)$ defined in terms of the kernels of the maps

$$
\pi_{*}(X) \longrightarrow \pi_{*}\left(L_{n} X\right) \text {. }
$$

The chromatic convergence theorem (1.2) says that the inverse limit is $X$ when $X$ is a $p$-local finitecomplex. $L_{0} X$ is the rational homotopy type of $X$ and $L_{1} X$ is its localization with respect to $p$-local complex $\mathrm{K}$-theory. Both of these functors are well understood. The other functors are less familiar but manageable, and we have a good handle on the $n^{\text {th }}$ 'layer' of the chromatic tower, i.e., the fibre of the map

$$
L_{n} X \longrightarrow L_{n-1} X .
$$

*Partially supported by the National Science Foundation 
Thomason's theorem (3.1) says that when $X$ is the K-theory spectrum for a reasonable ring $R$, then for each positive $i, \pi_{i}\left(L_{1} X\right)$ is precisely the value of $K_{i}(R)$ predicted by the Lichtenbaum-Quillen conjectures. Mitchell's theorem (3.2) says that in this case $L_{n} X=L_{1} X$ for all $n>1$, i.e., the higher layers of the tower tell us nothing about conventional algebraic K-theory.

However, the chromatic filtration is of more interest in Waldhausen's algebraic K-theory of spaces as explained in [Wal84]. Very recently, McClure and Staffeldt [MSb] have proved a convergence theorem for one Waldhausen's versions of the chromatic tower.

In Section 1 we will give a very expository account of the chromatic filtration; a much fuller account can be found in [Rav92a].

In Section 2 we will define some new localization functors $L_{n}^{f}(2.6)$, which leads to a new chromatic filtration suggested by the failure of the telescope conjecture. It has most of the desirable properties of the original one, with the exception of the chromatic convergence theorem, which is still an open question. We use the notation $L_{n}^{f}$ (suggested by Miller) to emphasize the connection with finite complexes; this functor is finite (see Definition 1.7) in the sense of Waldhausen [Wal84]. The map $X \rightarrow L_{n} X$ factors canonically through $L_{n}^{f} X$, but the explicit relation between the two functors is still mystery.

The results of this section (and also Corollary 1.19) are new and we will give detailed proofs. They do not depend logically on our disproof of the telescope conjecture. These results have been obtained independently by MahowaldSadofsky [MSa] and Miller [Mil].

In Section 3 we will describe some connections with algebraic K-theory, namely the theorems of Thomason (Theorem 3.1) and Mitchell (Theorem 3.2), Waldhausen's program and the convergence theorem recently proved by McClureStaffeldt (Theorem 3.7). I am grateful to Mitchell, McClure, Staffeldt and Waldhausen for helpful discussions on this material.

Throughout this paper all spectra will be assumed to be p-local for a fixed prime $p$ unless otherwise stated.

\section{The chromatic filtration}

Before giving the definitions needed for the chromatic filtration, we will discuss why it is interesting. In the diagram

$$
L_{0} X \longleftarrow L_{1} X \longleftarrow L_{2} X \longleftarrow \cdots
$$

the functors $L_{n}$ are Bousfield localizations, rather mysterious objects whose existence and properties are guaranteed by a deep result of Bousfield (Theorem 1.5). The original motivation for studying them comes from the properties of the Adams-Novikov spectral sequence described first in [MRW77], and later in [Rav86] and [Rav92a]. Fortunately we need not discuss these computational intricacies here. Suffice it to say the following: 
- Let $M_{n} X$ denote the fibre of the map $L_{n} X \rightarrow L_{n-1} X$, the ' $n$th layer' of the chromatic filtration. There are effective methods for computing its homotopy groups completely in some interesting cases.

- The graded group $\pi_{*}\left(M_{n} X\right)$ is always organized in a particular way. Its elements fall into ' $v_{n}$-periodic families;' for each nontrivial $x \in \pi_{s}\left(M_{n} X\right)$ there is a $j$ such that there is a corresponding nontrivial element in $\pi_{t}\left(M_{n} X\right)$ for each $t$ congruent to $s$ modulo $2 p^{j}\left(p^{n}-1\right)$.

There is also the following convergence theorem, proved in [HRa] and in [Rav92a, §8.6].

Theorem 1.2 (Chromatic convergence theorem) For a p-local finite complex $X$,

$$
X \simeq \lim _{\leftarrow} L_{n} X .
$$

\section{Localization}

Now we need to discuss the localization functors $L_{n}$ more precisely. In the simplest sense, localization at a prime $p$ means 'ignoring torsion prime to $p$.' In algebraic topology this translates into ignoring phenomena which can not be detected by mod $p$ homology. (We will make this more precise momentarily.) In the 1960's the concept of homology itself was generalized substantially. We now associate a generalized homology theory to a spectrum $E$ by defining

$$
E_{*}(X)=\pi_{*}(E \wedge X) .
$$

Here $X$ can be either a space or a spectrum. In the case of ordinary homology, the spectrum $E$ is made of Eilenberg-Mac Lane spaces.

Localizing with respect to $E$ means ignoring phenomena not detected by $E$-homology. One could do this formally by taking the homotopy category and formally inverting all maps inducing isomorphisms in $E$-homology; this is the approach taken by Gabriel and Zisman in [GZ67]. One then has a 'category of fractions' in which the objects are not spaces or spectra but equivalence classes of same. Bousfield's theorem says that this artificial category can in fact be embedded as a full subcategory in the original category. Without proving the theorem, it is easy to say what the objects in the embedded category should be.

Definition 1.3 (i) A space or spectrum $X$ is E-local if for every map

$$
W_{1} \stackrel{f}{\longrightarrow} W_{2}
$$

with $E_{*}(f)$ an isomorphism, the map

$$
\left[W_{1}, X\right] \stackrel{f^{*}}{\longleftarrow}\left[W_{2}, X\right]
$$

is an isomorphism. 
(ii) An E-localization of an arbitrary space or spectrum $X$ is a map

$$
X \stackrel{\lambda}{\longrightarrow} L_{E} X
$$

with $L_{E} X$ local as above and $E_{*}(\lambda)$ an isomorphism.

Thus $L_{E} X$, if it exists, is the image of $X$ under the composition of the Gabriel-Zisman construction and Bousfield's embedding. Once it has been constructed, we no longer need the Gabriel-Zisman construction.

The following properties of $L_{E} X$ are formal consequences of the definition.

Proposition 1.4 If the map $\lambda$ of 1.3(ii) exists, then:

(i) It is unique up to homotopy.

(ii) Given any map $f: X \rightarrow X^{\prime}$ with $E_{*}(f)$ an isomorphism, there is a unique (up to homotopy) map $\lambda^{\prime}$ making the following diagram commute

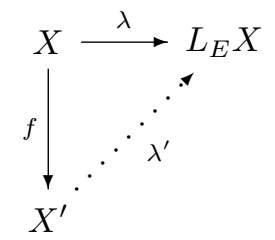

i.e., $\lambda$ is terminal among all $E_{*}$-equivalences out of $X$.

(iii) Given any map $g$ from $X$ to an E-local spectrum $Y$, there is a unique (up to homotopy) map $g^{\prime}$ making the following diagram commute

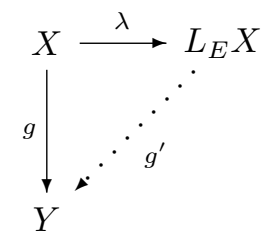

i.e., $\lambda$ is initial among all maps to E-local targets from $X$.

This result suggests that $L_{E} X$ can be constructed as the direct limit of all $E_{*}$-equivalences out of $X$. There are set theoretic difficulties with this, because the collection of all such maps forms a class rather than a set. Bousfield found a way around these problems and proved the following in [Bou75] and [Bou79].

Theorem 1.5 (Bousfield localization theorem) For any spectrum $E$ and any space or spectrum $X$, the localization $L_{E} X$ exists.

One can ask for additional properties of the functors $L_{E}$. 
Definition 1.6 A spectrum $E$ (or the functor $L_{E}$ ) is smashing if it has any of the following properties (which were shown to be equivalent in [Rav84, Prop. 1.27]).

(i) $L_{E}=L_{T}$ where $T=L_{E} S^{0}$.

(ii) $L_{E} X=X \wedge T$.

(iii) $L_{E}$ commutes with direct limits.

(iv) Any direct limit of E-local spectra is E-local.

The functors $L_{n}$ of 1.10 and $L_{n}^{f}$ of 2.6 are both smashing. The latter statement is easy, but the former is a deep theorem proved in [Rav92a, Chapter 8].

A still stronger condition on the functor $L_{E}$ is the following.

Definition 1.7 A localization functor $L_{E}$ is finite if there is a (possibly infinite) collection of finite spectra $M=\left\{X_{\alpha}\right\}$ such that the class of $E_{*}$-acyclic spectra is precisely the class of spectra obtainable from elements of $M$ by homotopy equivalences, direct limits, cofibres, desuspensions and (redundantly) retracts.

An equivalent definition was given by Waldhausen [Wal84, page 180]. We will explain now why the retract condition is redundant. Let $C$ be a class of spectra closed under homotopy equivalence, mapping cones, (possibly infinite) wedges, desuspension and (redundantly) suspensions. Now suppose that $W=X \vee Y$ is in $C$. Let $f: W \rightarrow W$ be the composite

$$
X \vee Y \rightarrow X \rightarrow X \vee Y .
$$

Then $X$ is the homotopy direct limit obtained by iterating $f$, i.e., the cofibre of a certain self map on the wedge of countably many copies of $W$. Hence $X$ is in $C$.

Bousfield proved that $L_{E}$ is smashing if it is finite, and he conjectured the converse. The functors $L_{n}^{f}$ are finite (almost by construction), but the statement that $L_{n}$ is finite is equivalent to the telescope conjecture, and therefore true for $n \leq 1$ but probably false for $n \geq 2$. Hence Bousfield's conjecture, like the telescope conjecture, is false. However we can say that the $L_{n}^{f}$ are the only (modulo the usual complications of mixing primes) finite localization functors. This is an easy consequence of the thick subcategory theorem. These assertions will be proved below in Corollary 1.19.

\section{$B P$ and related theories}

The functors $L_{n}$ used in the chromatic filtration are Bousfield localizations with respect to certain homology theories, which we need to discuss now. We will be very brief, referring the curious reader to [Rav92a], [Rav86] or [Rav84] 
and the references therein for more details. For each prime $p$ there is a ring spectrum $B P$ satisfying

$$
\pi_{*}(B P)=\mathbf{Z}_{(p)}\left[v_{1}, v_{2}, \cdots\right] \quad \text { where } \quad\left|v_{n}\right|=2 p^{n}-2 .
$$

The details of its construction need not concern us here. The following result, due to Devinatz, Hopkins and J. Smith [DHS88], is an indication of the importance of this homology theory.

Theorem 1.8 (Nilpotence theorem) Let $X$ be a finite complex and suppose we have a map $f: \Sigma^{d} X \rightarrow X$ for some $d \geq 0$. Then $f$ is nilpotent in the sense that some composite in the diagram

$$
X \longleftarrow \Sigma^{d} \Sigma^{d} X \stackrel{\Sigma^{d} f}{\longleftarrow} \Sigma^{2 d} X \stackrel{\Sigma^{2 d} f}{\longleftarrow} \Sigma^{3 d} X \stackrel{\Sigma^{3 d} f}{\longleftarrow} \ldots
$$

is null homotopic, if and only if the same is true of the homomorphism $B P_{*}(f)$.

In order to define $L_{n}$ for each $n \geq 0$, we need the spectrum $v_{n}^{-1} B P$, which is the direct limit of the diagram

$$
B P \stackrel{v_{n}}{\longrightarrow} \Sigma^{-\left|v_{n}\right|} B P \stackrel{v_{n}}{\longrightarrow} \Sigma^{-2\left|v_{n}\right|} B P \stackrel{v_{n}}{\longrightarrow} \cdots .
$$

(For $n=0$, we use $v_{0}=p$.) The map we are calling $v_{n}$ is constructed from $v_{n} \in \pi_{*}(B P)$ as follows. The latter is a map from $S^{\left|v_{n}\right|}$ to $B P$. We can smash both source and target with $B P$ and get a diagram

$$
\Sigma^{\left|v_{n}\right|} B P \stackrel{v_{n} \wedge B P}{\longrightarrow} B P \wedge B P \stackrel{m}{\longrightarrow} B P
$$

where $m$ is the multiplication map for the ring spectrum $B P$. The resulting composite is the map used in (1.9).

Definition 1.10 The functor $L_{n}$ of (1.1) is Bousfield localization with respect to $v_{n}^{-1} B P$.

The maps used in (1.1) are not obvious from what has been said here, but they are explained in [Rav84].

A closely related concept is the following.

Definition 1.11 For a spectrum $X, L_{\infty} X$ denotes $L_{E} X$ for

$$
E=\bigvee_{n \leq 0} v_{n}^{-1} B P
$$

$X$ is harmonic if $L_{\infty} X=X$ and dissonant if $L_{\infty} X=\mathrm{pt}$. 
In general a spectrum need be neither harmonic nor dissonant. There is always a cofibre sequence

$$
D \longrightarrow X \longrightarrow L_{\infty} X
$$

with $D$ dissonant and $L_{\infty} X$ harmonic.

In [Rav84, Theorem 4.4] it was shown that many spectra are harmonic. These include all finite spectra and all connective spectra with torsion free homology. On the other hand, the mod $p$ Eilenberg-Mac Lane spectrum is known to be dissonant. In $[\mathrm{HRb}]$ we showed that all suspension spectra are harmonic.

Note that $L_{\infty} X$ is not necessarily the same as $\lim _{\leftarrow} L_{n} X$. There is a natural map

$$
L_{\infty} X \longrightarrow \lim _{\leftarrow} L_{n} X
$$

and we have

$$
v_{m}^{-1} B P_{*}(X)=v_{m}^{-1} B P_{*}\left(L_{\infty} X\right)=\lim _{\leftarrow} v_{m}^{-1} B P_{*}\left(L_{n} X\right)
$$

for each $m$. However, since homology need not commute with inverse limits, we do not know in general that

$$
\lim _{\leftarrow} v_{m}^{-1} B P_{*}\left(L_{n} X\right)=v_{m}^{-1} B P_{*}\left(\lim _{\leftarrow} L_{n} X\right) .
$$

The chromatic convergence theorem says that this equality does hold for finite $X$.

\section{Morava K-theory and periodicity}

Closely related to $B P$ are the Morava K-theories. These are ring spectra (and $B P$-module spectra) $K(n)$ for $n \geq 0$ satisfying

$$
\pi_{*}(K(n))= \begin{cases}\mathbf{Q} & \text { if } n=0 \\ \mathbf{Z} /(p)\left[v_{n}, v_{n}^{-1}\right] & \text { if } n>0 .\end{cases}
$$

Each of these rings is a graded field in the sense that every graded module over it is free. $K(0)_{*}(X)$ is the same as the rational homology of $X$ and $K(1)_{*}(X)$ is one of $p-1$ isomorphic summands of the $\bmod p$ classical complex K-theory of $X$.

As we will see below, there is very little connection between the Morava Ktheories for $n>1$ and algebraic K-theory. It is also known that in some sense the Morava K-theories are independent of each other; there are no nontrivial natural transformations between them. On the other hand, there is some linkage between their values on a finite complex, as the following result illustrates.

Proposition 1.12 Let $X$ be a finite complex.

(i) The rank of $K(n)_{*}(X)$ (as a free module over $\pi_{*}(K(n))$ ) is no less than that of $K(n-1)_{*}(X)$. In particular, if $K(n)_{*}(X)=0$, then $K(n-$ $1)_{*}(X)=0$. 
(ii) $K(n)_{*}(X)=0$ if and only if $v_{n}^{-1} B P_{*}(X)=0$.

(iii) For large $n$, the rank of $K(n)_{*}(X)$ is the same as that of $H_{*}(X ; \mathbf{Z} /(p))$. In particular, if $K(n)_{*}(X)=0$ for all $n$ then $X$ is contractible.

This suggests the following definition.

Definition 1.13 A finite complex $X$ has type $n$ if $K(n-1)_{*}(X)=0$ and $K(n)_{*}(X) \neq 0$ (or equivalently if $v_{n-1}^{-1} B P_{*}(X)=0$ and $v_{n}^{-1} B P_{*}(X) \neq 0$ ).

Note that $X$ has type 0 unless $H_{*}(X ; \mathbf{Q})=0$, and one rarely encounters complexes of type greater than 1 . The existence of type $n$ complexes for all $n$ and $p$ was established by Mitchell in [Mit85].

Definition 1.14 A map $f: \Sigma^{d} X \rightarrow X$ is a $v_{n}$-map if $K(n)_{*}(f)$ is an isomorphism and $K(m)_{*}(f)=0$ for all $m \neq n$.

A major result in this area is the following, due to [HS]. A proof is also given in [Rav92a, Chapter 6].

Theorem 1.15 (Periodicity theorem) (i) For each finite complex $X$ of type at least $n$ there is a $v_{n}$-map $f: \Sigma^{d} X \rightarrow X$.

(ii) Given two such complexes $X_{1}$ and $X_{2}$ with $v_{n}$-maps $f_{1}$ and $f_{2}$ and any map $g: X_{1} \rightarrow X_{2}$, there are positive integers $i$ and $j$ such that the following diagram commutes up to homotopy

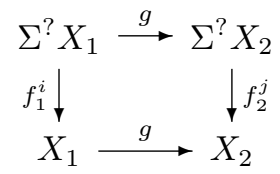

One might ask if there can be a $v_{m}$-map on a type $n$ complex for $m \neq n$. For $m<n$, the trivial map is a $v_{m}$-map so the question is uninteresting. For $m>n$ there are algebraic considerations (having to do with $B P$-theory) that preclude the existence of such a map.

Note that (ii) above is a uniqueness statement. It says (when $g$ is the identity map) that some iterate of $f_{1}$ is homotopic to some iterate of $f_{2}$.

Definition 1.16 Let $Y$ be a p-local finite complex of type $n$ with $v_{n}$-map $f$. Then the $v_{n}$-periodic homotopy with coefficients in $Y, v_{n}^{-1} \pi_{*}(X ; Y)$, of an arbitrary space or spectrum $X$ is the direct limit

$$
[Y, X]_{*} \stackrel{f^{*}}{\longrightarrow}\left[\Sigma^{d} Y, X\right]_{*} \stackrel{f^{*}}{\longrightarrow}\left[\Sigma^{2 d} Y, X\right]_{*} \stackrel{f^{*}}{\longrightarrow} \cdots .
$$

Note that 1.15(ii) implies that this group is independent of the choice of $f$. Typically one studies this group when $Y$ is as small as possible, i.e., when it is a complex with $2^{n}$ cells. For $n=0$ and $Y=S^{0}$, it is the rational homotopy of $X$ when $X$ is $p$-local. 
For $n=1$ and $Y$ is a $\bmod p^{i}$ Moore space, these groups have been studied by Mahowald [Mah82] and Thompson [Tho90] for $X$ a sphere, and by Bendersky, Davis , Mahowald and Mimura ([Ben92], [BD92], [BDM], [Dav91] and [DM92]) for $X$ a Lie group.

The question of whether a map $g: X_{1} \rightarrow X_{2}$ induces an isomophism in $v_{n^{-}}$ periodic homotopy depends only on $n$ and not on the choice of $Y$. If $v_{n}^{-1} \pi_{*}(g)$ is an isomorphism, then so is $K(n)_{*}(g)$. The converse of this statement (for all $X)$ is equivalent to the telescope conjecture, and is therefore true for $n \leq 1$ and probably false for all $n \geq 2$.

There is one other result we should mention. First we need a definition.

Definition 1.17 A full subcategory $\mathbf{C}$ of the category of finite spectra is thick if it satisfies the following two conditions.

(i) If

$$
W \longrightarrow X \longrightarrow Y \longrightarrow \Sigma W
$$

is a cofibre sequence and any two of $W, X$ and $Y$ are in $\mathbf{C}$, then so is the third.

(ii) If $X \vee Y$ is in $\mathbf{C}$ then so is $X$.

Examples of thick subcategories include the class $\mathbf{F}_{p, n}$ of $p$-local spectra trivialized by $v_{n-1}^{-1} B P$ or equivalently $K(n-1)$. Without the second condition of the definition, the class of spectra with vanishing Euler characteristic (however it is defined) would also qualify.

For the proof of the following result, see [HS, Theorem 7] (the original source), [Hop87], [Rav90, Theorem 2.19] or [Rav92a, Theorem 3.4.3].

Theorem 1.18 (Thick subcategory theorem) Let $\mathbf{C}$ be a nontrivial thick subcategory of $\mathbf{F}_{(p)}$, the category of p-local finite $C W$-spectra. Then $\mathbf{C}=\mathbf{F}_{p, n}$ for some $n$. (Note that $\mathbf{F}_{p, 0}$ is all of $\mathbf{F}_{(p)}$.)

This result is extremely useful. For example the periodicity theorem is proved by showing first that the collection of finite spectra admitting $v_{n}$-maps is thick. This means that it is either $\mathbf{F}_{p, n}$ or $\mathbf{F}_{p, n+1}$. (If $K(n)_{*}(X)$ is trivial for a finite complex $X$, then the trival map on $X$ is a $v_{n}$-map.) Hence it suffices to construct a single nontrivial example of a finite complex with a $v_{n}$-map. This was done by Jeff Smith in [Smi].

Corollary $\mathbf{1 . 1 9}$ (i) The functors $L_{n}^{f}$ (to be defined below in 2.6) are finite in the sense of $1 . \%$.

(ii) The $L_{n}^{f}$ are the only finite localization functors on the category of p-local spectra.

(iii) The functor $L_{n}$ is finite if and only if the telescope conjecture (2.2) is true for the given values of $p$ and $n$. 
Proof. (i) The $L_{n}^{f}$ are finite because the class of $p$-local $L_{n}^{f}$-acyclics is generated by a finite complex of type $n+1$.

(ii) A finite $p$-local localization functor is determined by the class $p$-local finite spectra which it trivializes. This class must be thick, so it must be $\mathbf{F}_{p, n}$ for some $n$, and the functor must be $L_{n}^{f}$.

(iii) If $L_{n}^{f}=L_{n}$ (as asserted by the telescope conjecture) then $L_{n}$ is also finite. Conversely, suppose that $L_{n}$ is finite. Then it must be the same as $L_{n}^{f}$ by (ii), and the telescope conjecture follows.

\section{An alternate definition of the chromatic filtra- tion}

In this section we will define functors $L_{n}^{f}$ (Definition 2.6) which are finite in the sense of 1.7. This construction was discovered independently by MahowaldSadofsky [MSa] and Miller [Mil]. First we need a definition.

Definition 2.1 Given a type $n$ complex $X$ with $v_{n}$-map $f$, the associated $v_{n}$ telescope $\widehat{X}$ is the homotopy direct limit of the diagram

$$
X \stackrel{f}{\longrightarrow} \Sigma^{-d} X \stackrel{f}{\longrightarrow} \Sigma^{-2 d} X \stackrel{f}{\longrightarrow} \cdots
$$

It follows from 1.15(ii) that $\widehat{X}$ is independent of the choice of $f$. In fact the directed system of 2.1 is cofinal in the set of all $v_{n}$-maps out of $X$.

For the record we restate the following, even though it is no longer viable.

Conjecture 2.2 (Telescope conjecture) For $X$ as in 2.1, the map $\widehat{X} \rightarrow$ $L_{n} X$ is an equivalence.

We now know that this is false for $n=2$, and presumably for all $n>2$. Our new functor $L_{n}^{f}$ will have the property $(2.7(\mathrm{v}))$ that for $X$ as above, $\widehat{X}=L_{n}^{f} X$.

Now we can describe our method for constructing $L_{n}^{f}$. We will see below (2.7) that it suffices to define $L_{n}^{f} S^{0}$, which we do by induction on $n$. We start with $L_{0}^{f} S^{0}=\widehat{S}^{0}$, which is the direct limit of

$$
S^{0} \stackrel{p}{\longrightarrow} S^{0} \stackrel{p}{\longrightarrow} S^{0} \stackrel{p}{\longrightarrow} \cdots .
$$

Let $C_{0}^{f}$ denote the cofibre of the map $S^{0} \rightarrow L_{0}^{f} S^{0}$. It is the direct limit of finite spectra of type 1, namely

$$
S^{1} / p \longrightarrow S^{1} / p^{2} \longrightarrow S^{1} / p^{3} \longrightarrow \cdots .
$$

There is a corresponding limit of $v_{1}$-telescopes

$$
\widehat{S^{1} / p} \longrightarrow \widehat{S^{1} / p^{2}} \longrightarrow \widehat{S^{1} / p^{3}} \longrightarrow \cdots,
$$

which we denote by $\widehat{C}_{0}^{f}$. 
Now we define $L_{1}^{f} S^{0}$ to be the fibre of the map $L_{0}^{f} S^{0} \rightarrow \widehat{C}_{0}^{f}$. Then we have the following commutative diagram in which each row and column is a cofibre sequence.

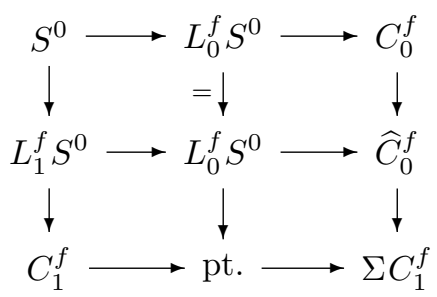

where $C_{1}^{f}$ is the evident cofibre. It is a direct limit of finite complexes of type 2 .

Inductively, suppose that $L_{n-1}^{f} S^{0}$ and $C_{n-1}^{f}$ have been defined and that the latter is a direct limit of finite complexes of type $n$. We will see below (Lemma 2.4 ) that there is a corresponding $v_{n}$-telescope $\widehat{C}_{n-1}^{f}$ and a similar diagram

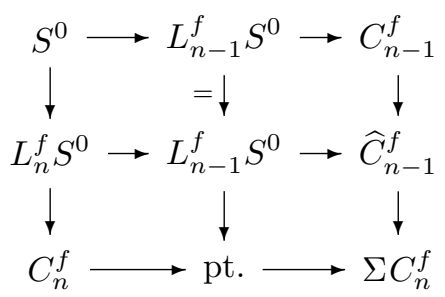

In order to make this work, we need the following lemma, which says roughly that 'telescoping commutes with direct limits.'

Lemma 2.4 (i) Given a map $g: X_{1} \rightarrow X_{2}$ of type $n$ finite complexes, there is a canonical map of $v_{n}$-telescopes $\widehat{g}: \widehat{X}_{1} \rightarrow \widehat{X}_{2}$.

(ii) For a type $n$ finite complex $X$, let $C X$ denote the cofibre of the map $X \rightarrow \widehat{X}$. Then $C X$ is a direct limit $\lim _{\rightarrow} X_{\alpha}$ of finite complexes of type $n+1$ that is locally finite, i.e., for each index $\beta$ there are only finitely many indices $\alpha$ with $\alpha<\beta$.

(iii) Let $\lim _{\rightarrow} X_{\alpha}$ denote a locally finite direct limit of finite complexes of type $n$ in which the indexing set has a cofinal subset isomorphic to the natural numbers, and let $C \lim _{\rightarrow} X_{\alpha}$ denote the cofibre of the map $\lim _{\rightarrow} X_{\alpha} \rightarrow$ $\lim _{\rightarrow} \widehat{X}_{\alpha}$. Then $C \lim _{\rightarrow} \widehat{X}_{\alpha}$ is a locally finite limit of finite complexes of type $n+1$ whose indexing set has the same property.

Proof. (i) It follows from 1.15 (ii) that $X_{1}$ and $X_{2}$ have $v_{n}$-maps $f_{1}$ and $f_{2}$ such that $g f_{1}=f_{2} g$, and the iterates of $f_{j}$ form a cofinal subset of all the $v_{n}$-maps out 
of $X_{j}$ for each $j$. Hence we get a commutative diagram (ignoring suspensions)

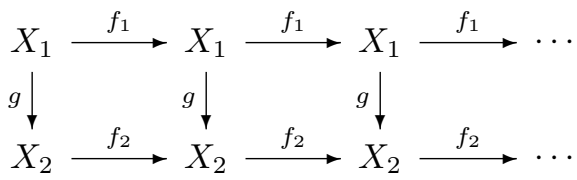

which gives the desired map $\widehat{g}$.

(ii) Choose a $v_{n}$-map $f$ on $X$ and consider the diagram (again ignoring suspensions)

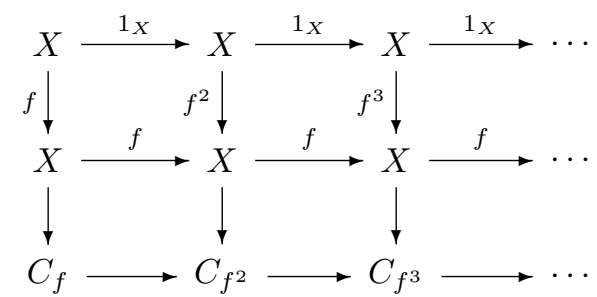

where $C_{f^{i}}$ denotes the cofibre of $f^{i}$. Then each complex in the bottom row is finite of type $n+1$, and the (locally finite) limit is $C X$.

(iii) We can use (i) to construct a map from $\lim _{\rightarrow} X_{\alpha}$ to $\lim _{\rightarrow} \widehat{X}_{\alpha}$. We would like to say that

$$
C \lim _{\rightarrow} \widehat{X}_{\alpha}=\lim _{\rightarrow} C X_{\alpha}
$$

but the map $C g_{\alpha \beta}: C X_{\alpha} \rightarrow C X_{\beta}$ for $\alpha<\beta$ is not uniquely determined by $g_{\alpha \beta}$ and $\widehat{g}_{\alpha \beta}$. However, if we use the cofinal subset given by hypothesis, we can choose maps $C g_{\alpha \beta}$ without having to worry about any diagrams commuting. Hence we can define $\lim _{\rightarrow} C X_{\alpha}$. It is equivalent to $C \lim _{\rightarrow} \widehat{X}_{\alpha}$ because homotopy direct limits preserve cofibre sequences, and therefore independent of the choice of $C g_{\alpha \beta}$.

Now each $C X_{\alpha}$ is a limit with the desired properties by (ii), so the same is true of $\lim _{\rightarrow} C X_{\alpha}$ and hence of $C \lim _{\rightarrow} \widehat{X}_{\alpha}$.

Corollary 2.5 The construction of $L_{n}^{f} S^{0}$ of (2.3) is well defined.

We are now ready for our main definition.

Definition 2.6 The functor $L_{n}^{f}$ is localization with respect to the spectrum $L_{n}^{f} S^{0}$ of (2.3).

These functors enjoy the following properties.

Theorem 2.7 (i) When the functor $L_{n}^{f}$ is applied to $S^{0}$, the spectrum obtained is the one called in $L_{n}^{f} S^{0}$ in (2.3).

(ii) (Smash product theorem) For any spectrum $X, L_{n}^{f} X=X \wedge L_{n}^{f} S^{0}$. 
(iii) (Localization theorem) There is a natural transformation $L_{n}^{f} \rightarrow L_{n}$, with $L_{n}^{f} X \rightarrow L_{n} X$ a $B P_{*}$-equivalence for any $X$.

(iv) (Telescope theorem) For a finite spectrum $X$ of type $n, \widehat{X}=L_{n}^{f} X$.

We do not have a proof of the analog of the chromatic convergence theorem for the $L_{n}^{f}$; this is still an open question. Hal Sadofsky has pointed out that $X$ is a retract of the inverse limit since the identity map can be factored as

$$
X \longrightarrow \lim _{\leftarrow} L_{n}^{f} X \longrightarrow \lim _{\leftarrow} L_{n} X=X
$$

for any finite $X$.

Thus the $L_{n}^{f}$ have some of the nice properties of the $L_{n}$. One difference other than the absence of a chromatic convergence theorem is that the Adams-Novikov spectral sequence does not converge in general for $L_{n}^{f} X$, so $\pi_{*}\left(L_{n}^{f} X\right)$ is harder to compute. In view of the $B P_{*}$-equivalence of 2.7 (iii), this spectral sequence coincides with the one for $L_{n} X$, which was shown to converge in [Rav87]. Hence convergence of the Adams-Novikov spectral sequence for $L_{n}^{f} X$ for $X$ a type $n$ finite complex is equivalent to the telescope conjecture.

Before we can prove 2.7, we need the following.

Lemma 2.8 (i) Suppose $X$ is a type $m$ complex with $v_{m}$-telescope $\widehat{X}$ as in 2.1, and $Y$ is a complex of type $n$ with $m<n$. Then $\widehat{X} \wedge Y$ is contractible.

(ii) $L_{n}^{f} S^{0} \wedge L_{n}^{f} S^{0} \cong L_{n}^{f} S^{0}$.

Proof. (i) There are two evident $v_{m}$-maps on $X \wedge Y$, namely $f \wedge Y$ and the trivial map. Hence 1.15(ii) implies that some iterate of $f \wedge Y$ is null and it follows that $\widehat{X} \wedge Y$ is contractible.

(ii) Consider the cofibre sequence

$$
S^{0} \longrightarrow L_{n}^{f} S^{0} \longrightarrow C_{n}^{f}
$$

Smashing it with $L_{n}^{f} S^{0}$, we see that the result will follow if $L_{n}^{f} S^{0} \wedge C_{n}^{f}$ is contractible. By 2.4(iii), $C_{n}^{f}$ is a direct limit of finite complexes of type $n+1$. We will show that $L_{m}^{f} S^{0} \wedge C_{n}^{f}$ is contractible for $m \leq n$ by induction on $m$. For $m=0$ it follows from (i) since $L_{0}^{f} S^{0}$ is a $v_{0}$-telescope.

For the inductive step, by (2.3) we have a cofibre sequence

$$
L_{m}^{f} S^{0} \longrightarrow L_{m-1}^{f} S^{0} \longrightarrow \widehat{C}_{m-1}^{f}
$$

Then $L_{m-1}^{f} S^{0} \wedge C_{n}^{f}$ is contractible by the inductive hypothesis and $\widehat{C}_{m-1}^{f} \wedge C_{n}^{f}$ is contractible by $2.4($ iii) and (i).

Proof of Theorem 2.7. (i) To avoid confusion, let $E$ denote the spectrum $L_{n}^{f} S^{0}$ of (2.3). By Lemma 2.8(ii)), $E \wedge E=E$. This means that $E$ is a ring spectrum, with the unit map $\eta: S^{0} \rightarrow E$ being given in (2.3). It follows that the unit map $\eta$ is an $E_{*}$-equivalence. Therefore if $E$ is $E_{*}$-local then $E=L_{E} S^{0}$ as claimed. 
However, any ring spectrum $E$ is local with respect to itself [Rav84, 1.17]. To see this, observe that any map $f: X \rightarrow E$ can be factored as follows.

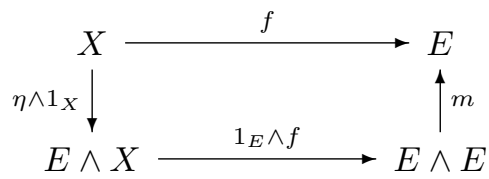

where $\eta$ and $m$ are the unit and multiplication maps for $E$. Therefore if $E_{*}(X)=$ 0 , the map $f$ is null homotopic, so $E$ is local by definition (1.3). (A similar argument shows that any $E$-module spectrum is $E_{*}$-local; this will be needed in (ii) below.)

(ii) In $[\operatorname{Rav} 84,1.29]$ it was shown that $L_{E} X=X \wedge L_{E} S^{0}$ for any ring spectrum $E$ with $E \wedge E=E$. To see this, note that the map $\eta \wedge 1_{X}: X \rightarrow E \wedge X$ is an $E_{*}$-equivalence whose target is an $E_{*}$-module spectrum and therefore $E_{*}$ local.

(The proof of the original smash product theorem, which says that $L_{n} X=$ $X \wedge L_{n} S^{0}$, can be found in [Rav92a, Chapter 8]. In contrast to the argument just given, it is quite long. If the telescope conjecture, which says that $L_{n}^{f}=L_{n}$, were true, then the smash product theorem would be an easy consequence.)

(iii) Given that both $L_{n}^{f}$ and $L_{n}$ satisfy the smash product theorem, it suffices to construct a suitable map $L_{n}^{f} S^{0} \rightarrow L_{n} S^{0}$ which is a $B P_{*}$-equivalence. In view of (2.3) it suffices to construct a diagram

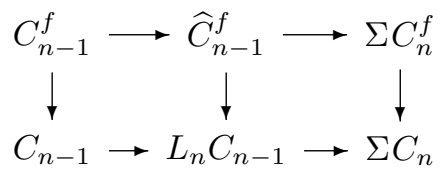

(where $C_{n}$ is the cofibre of $S^{0} \rightarrow L_{n} S^{0}$ ) in which each vertical map is a $B P_{*}$ equivalence. It is understood that $C_{-1}^{f}=C_{-1}=S^{1}$. We will argue by induction on $n$. For $n=0$ the left hand vertical map is the identity, as is the middle one, so the right hand one is also.

For the inductive step, assume that the left hand map is defined and is a $B P_{*}$-equivalence. This means that $L_{n} C_{n-1}=L_{n} C_{n-1}^{f}$. Now $C_{n-1}^{f}$ is a limit of finite complexes of type $n$, so both maps out of it are $v_{n}^{-1} B P_{*}$-equivalences. This means there is a unique choice for the middle vertical map. We know by the main result of [Rav87] that

$$
B P_{*}\left(L_{n} C_{n-1}\right)=v_{n}^{-1} B P_{*}\left(C_{n-1}\right)
$$

It is straightforward that $B P_{*}\left(\widehat{C}_{n-1}^{f}\right)$ has the same description and that the middle map is a $B P_{*}$-equivalence. It follows that the same is true of the right hand vertical map.

(iv) Let $C_{m}^{f} X$ denote the cofibre of $X \rightarrow L_{m}^{f} X$. Smashing $X$ with the right hand column of (2.3) gives us a cofibre sequence

$$
C_{m-1}^{f} X \longrightarrow X \wedge \widehat{C}_{m-1}^{f} \longrightarrow \Sigma C_{m}^{f} X
$$


If $X$ is a finite complex of type $n$, then by 2.4(iii) and 2.8(i), the middle term is contractible for $m<n$. It follows that $C_{n-1}^{f} X$ is $\Sigma X$ and $L_{n-1}^{f} X$ is contractible. Thus smashing the middle row of (2.3) with $X$ gives the desired cofibre sequence

$$
L_{n}^{f} X \longrightarrow \text { pt. } \longrightarrow \Sigma \widehat{X}
$$

\section{Connections with algebraic K-theory}

In this section we shall recall some results of Thomason, Mitchell, Waldhausen and McClure-Staffeldt which indicate the relevance of the chromatic filtration to algebraic K-theory. For background on the first half of this material, we recommend the paper of Mitchell [Mitb]. We begin with a rough statement of the theorem of Thomason [Tho85], reinterpreted by Waldhausen in [Wal84] and quoted as Theorems 7.10 and 11.4 in [Mitb].

Theorem 3.1 Let $R$ be a nice ring. If $p=2$, assume that $\sqrt{-1} \in X$. Then for $i$ sufficiently large ( $i \geq 1$ when $R$ is the ring of integers in a number field), $\pi_{i}\left(L_{1} K R\right)$ is the value of $K_{i} R$ given by the Lichtenbaum-Quillen conjectures.

This statement is admittedly vague, and we refer the reader to [Mitb] for the details. The precise meaning of 'nice' is given in [Tho85]; it is a very mild condition on $R$. (One can replace rings by schemes throughout, but we leave that to the cognescenti.) The point is that the Lichtenbaum-Quillen conjectures can be reformulated in chromatic terms. They say that the spectrum $K R$ differs from a connective cover of $L_{1} K R$ by a finite Postnikov system, with homotopy concentrated in dimension zero when $R$ is a suitable ring of integers.

As explained by Mitchell in [Mitb, §11], a consequence of this reformulation is the following result, which he proved in [Mit90]. It is Theorem 12.4 of [Mitb].

Theorem 3.2 For any ring $R, K(n)_{*}(K R)=0$ for all $n \geq 2$.

This means that $L_{n} K R=L_{1} K R$ for all $n \geq 1$. It also says that higher Morava K-theory and higher algebraic K-theory (in the sense of Quillen) have nothing to do with each other.

Corollary 3.3 ([Mita]) For any ring $R, L_{\infty} K R$ (see 1.11) is the same as $L_{1} K R$. Moreover, $L_{\infty} K R$ is the same as the inverse limit $\lim _{\leftarrow} L_{n} K R$.

In particular the Lichtenbaum-Quillen conjectures hold for $L_{\infty} K R$ for nice $R$, and the fibre of the map $K R \rightarrow L_{1} K R$ (i.e., the error term) is dissonant (Definition 1.11).

What Mitchell proved is actually stronger than Theorem 3.2. The following is essentially [Mit90, Theorem 3.8]. 
Theorem 3.4 For any ring $R$ and all $n \geq 2$, the $v_{n}$-periodic homotopy (see 1.16) of $K R$ is trivial.

This means that we can make similar statements about $K R$ in terms of the functors $L_{n}^{f}$ defined in 2.6 .

Corollary 3.5 For each ring $R$ and each $n \geq 1, L_{n}^{f} K R=L_{1} K R$.

We now turn to Waldhausen's program, described in [Wal84]. A brief description can also be found in [MSb]. He defines two spectra, $\tilde{A}(*)$ and $A(*)$ (which are not $p$-local) and a natural map from the former to the latter, roughly as follows. The former, $\tilde{A}(*)$ is a generalization of Quillen's definition of $K R$ to the 'brave new ring' $Q S^{0}$. (The latter is known to be a ring object in the homotopy category, with addition corresponding to the loop space structure, and multiplication corresponding to composition of maps between spheres.) The latter, $A(*)$, is the geometric realization of a certain bisimplicial set based on the category of finite spectra, in which weak equivalences play a special role in the construction.

Now fix a prime $p$, and define $\tilde{A}(*, p, n)$ and $\tilde{A}^{f}(*, p, n)$ in a similar way with the sphere spectrum replaced by $L_{n} S^{0}$ and $L_{n}^{f} S^{0}$ respectively. (For $n=0$, Waldhausen deviates slightly from this. He replaces $L_{0} S^{0}=L_{0}^{f} S^{0}=H \mathbf{Q}$, the rational Eilenberg-Mac Lane spectrum, by $H \mathbf{Z}_{(p)}$, the $p$-local integral one. This means that $\tilde{A}^{f}(*, p, 0)=\tilde{A}(*, p, 0)=K \mathbf{Z}_{(p)}$, the algebraic K-theory of the $p$-local integers.) This leads to a commutative diagram

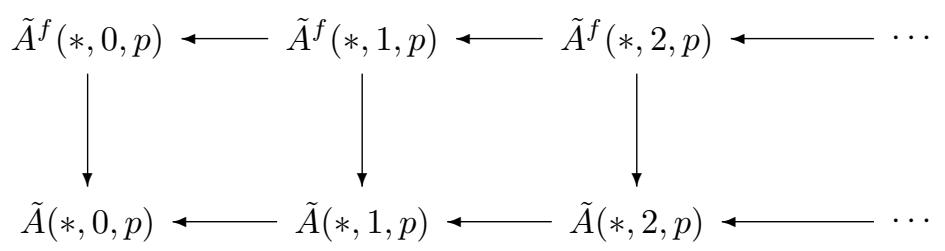

in which each map is induced by a natural transformation of localization functors. Waldhausen calls each row an integral localization tower. He does not distinguish between the two of them because he assumes (on page 187) that $L_{n}=L_{n}^{f}$, i.e., he assumes the telescope conjecture. We know that the vertical maps are equivalences for $n=0$ and $n=1$, but not for $n>1$.

One can also define spectra $A(*, p, n)$ in terms of the category of finite spectra, with weak equivalences replaced by $K(n)_{*}$-equivalences. Waldhausen's definition of the map $\tilde{A}(*) \rightarrow A(*)$ leads to a diagram

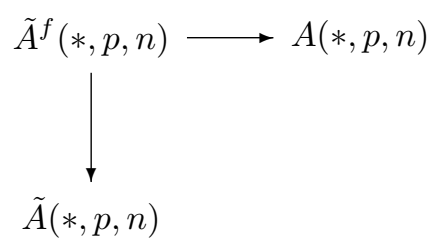


for each $n$ and $p$, but the horizontal map need not factor through $\tilde{A}(*, p, n)$.

The convergence theorem of McClure-Staffeldt [MSb], which is proved using our chromatic convergence theorem (1.2), is the following.

Theorem 3.7 Let $A(*, p)$ denote the localization of $A(*)$ at $p$. Then with notation as above,

$$
A(*, p)=\lim _{\leftarrow} \tilde{A}(*, p, n) .
$$

The convergence of the other two towers (obtained by varying $n$ ) in (3.6) is still an open question.

\section{References}

[BD92] M. Bendersky and D. M. Davis. 2-primary $v_{1}$-periodic homotopy groups of $S U(n)$. American Journal of Mathematics, 114:465-494, 1992.

[BDM] M. Bendersky, D. M. Davis, and M. Mimura. $v_{1}$-periodic homotopy groups of exceptional Lie groups - torsion-free cases. To appear in Transactions of the American Mathematical Society.

[Ben92] M. Bendersky. The $v_{1}$-periodic unstable Novikov spectral sequence. Topology, 31:47-64, 1992.

[Bou75] A. K. Bousfield. The localization of spaces with respect to homology. Topology, 14:133-150, 1975.

[Bou79] A. K. Bousfield. The localization of spectra with respect to homology. Topology, 18:257-281, 1979.

[Dav91] D. M. Davis. The $v_{1}$-periodic homotopy groups of $S U(n)$ at odd primes. Proceedings of the London Mathematical Society (3), 43:529$544,1991$.

[DHS88] E. Devinatz, M. J. Hopkins, and J. H. Smith. Nilpotence and stable homotopy theory. Annals of Mathematics, 128:207-242, 1988.

[DM92] D. M. Davis and M. E. Mahowald. Some remarks on $v_{1}$-periodic homotopy groups. In N. Ray and G. Walker, editors, Adams Memorial Symposium on Algebraic Topology Volume 2, pages 55-72, Cambridge University Press, Cambridge, 1992.

[GZ67] P. Gabriel and M. Zisman. Calculus of fractions and homotopy theory. Springer-Verlag, New York, 1967.

[Hop87] M. J. Hopkins. Global methods in homotopy theory. In J. D. S. Jones and E. Rees, editors, Proceedings of the 1985 LMS Symposium on Homotopy Theory, pages 73-96, 1987. 
[HRa] M. J. Hopkins and D. C. Ravenel. A proof of the smash product conjecture. To appear.

[HRb] M. J. Hopkins and D. C. Ravenel. Suspension spectra are harmonic. To appear in Bol. Soc. Math. Mexicana.

[HS] M. J. Hopkins and J. H. Smith. Nilpotence and stable homotopy theory II. Submitted to Annals of Mathematics.

[Mah82] M. E. Mahowald. The image of $\mathrm{J}$ in the EHP sequence. Annals of Mathematics, 116:65-112, 1982.

[Mil] H. R. Miller. Finite localizations. To appear.

[Mita] S. A. Mitchell. Harmonic localization of algebraic $K$-theory spectra. To appear in Transactions of the American Mathematical Society.

[Mitb] S. A. Mitchell. On the Lichtenbaum-Quillen conjectures from a stable homotopy-theoretic viewpoint. To appear in Topics in Algebraic Topology and its Applications, MSRI publication series, Springer Verlag (1992).

[Mit85] S. A. Mitchell. Finite complexes with $A(n)$-free cohomology. Topology, 24:227-248, 1985.

[Mit90] S. A. Mitchell. The Morava $K$-theory of algebraic $K$-theory spectra. K-Theory, 3:607-626, 1990.

[MRW77] H. R. Miller, D. C. Ravenel, and W. S. Wilson. Periodic phenomena in the Adams-Novikov spectral sequence. Annals of Mathematics, 106:469-516, 1977.

[MSa] M. E. Mahowald and H. Sadofsky. $v_{n}$-telescopes and the Adams spectral sequence. To appear.

[MSb] J. E. McClure and R. E. Staffeldt. The chromatic convergence theorem and a tower in algebraic $K$-theory. To appear in Proceedings of the American Mathematical Society.

[Rav] D. C. Ravenel. A counterexample to the telescope conjecture. To appear.

[Rav84] D. C. Ravenel. Localization with respect to certain periodic homology theories. American Journal of Mathematics, 106:351-414, 1984.

[Rav86] D. C. Ravenel. Complex Cobordism and Stable Homotopy Groups of Spheres. Academic Press, New York, 1986.

[Rav87] D. C. Ravenel. The geometric realization of the chromatic resolution. In W. Browder, editor, Algebraic topology and algebraic K-theory, pages 168-179, 1987. 
[Rav90] D. C. Ravenel. The nilpotence and periodicity theorems in stable homotopy theory. Astérisque, 189-190:399-428, 1990.

[Rav92a] D. C. Ravenel. Nilpotence and periodicity in stable homotopy theory. Volume 128 of Annals of Mathematics Studies, Princeton University Press, Princeton, 1992.

[Rav92b] D. C. Ravenel. Progress report on the telescope conjecture. In N. Ray and G. Walker, editors, Adams Memorial Symposium on Algebraic Topology Volume 2, pages 1-21, Cambridge University Press, Cambridge, 1992.

[Smi] J. Smith. Finite complexes with vanishing lines of small slope. To appear.

[Tho85] R. W. Thomason. Algebraic K-theory and étale cohomology. Ann. Scient. Écoles Norm. Sup., 13:437-552, 1985.

[Tho90] R. J. Thompson. Unstable $v_{1}$-periodic homotopy at odd primes. Transactions of the American Mathematical Society, 319:535-559, 1990.

[Wal84] F. Waldhausen. Algebraic $K$-theory of spaces, localization, and the chromatic filtration of stable homotopy theory. In I. Madsen and R. Oliver, editors, Algebraic Topology Aarhus 1982, pages 173-195, Lecture Notes in Mathematics, 1051, Springer-Verlag, 1984. 